\title{
Physical, chemical and biological sensors: innovative platforms for various industries
}

Mirko Lehmann

Innovative Sensor Technology IST AG

Stegrütistrasse 14 | 9642 Ebnat-Kappel | Switzerland

Mirko.lehmann@ist-ag.com

\begin{abstract}
Different physical, chemical and biological sensor elements and their role in the applications are described. Challenges in the development and the design-ins are very different when dealing with physical compared to biological sensors, some examples are given for that. And in regard to applications: What are the essential things to consider when dealing with different performance requests coming from different industries or the same industry? Furthermore a special view on innovations and a reflection on the importance of developing more sensor technologies will be given to enable sensor industry growth also in the future.
\end{abstract}

